\title{
Downregulation of membrane complement inhibitors CD55 and CD59 by siRNA sensitises uterine serous carcinoma overexpressing Her2/neu to complement and antibody-dependent cell cytotoxicity in vitro: implications for trastuzumab-based immunotherapy
}

\author{
S Bellone', D Roque', E Cocco', S Gasparrini', I Bortolomai', N Buza'2, M Abu-Khalaf', D-A Silasi', E Ratner', \\ M Azodi', PE Schwartz', TJ Rutherford', S Pecorelli' ${ }^{4}$ and AD Santin ${ }^{*},{ }^{\prime}$ \\ 'Department of Obstetrics, Gynecology and Reproductive Sciences, Yale University School of Medicine, New Haven, CT, USA; ${ }^{2}$ Department of Pathology, \\ Yale University School of Medicine, New Haven, CT, USA; ${ }^{3}$ Department of Internal Medicine and Oncology, Yale University School of Medicine, \\ New Haven, CT, USA; ${ }^{4}$ Division of Gynecologic Oncology, University of Brescia, Brescia, Italy
}

BACKGROUND: We evaluated the expression of CD46, CD55 and CD59 membrane-bound complement-regulatory proteins (mCRPs) in primary uterine serous carcinoma (USC) and the ability of small interfering RNA (siRNA) against these mCRPs to sensitise USC to complement-dependent cytotoxicity (CDC) and antibody (trastuzumab)-dependent cellular cytotoxicity (ADCC) in vitro.

METHODS: Membrane-bound complement-regulatory proteins expression was evaluated using real-time PCR (RT-PCR) and flow cytometry, whereas Her2/neu expression and c-erbB2 gene amplification were assessed using immunohistochemistry, flow cytometry and fluorescent in-situ hybridisation. The biological effect of siRNA-mediated knockdown of mCRPs on HER2/neu-overexpressing USC cell lines was evaluated in CDC and ADCC 4-h chromium-release assays.

RESULTS: High expression of MCRPs was found in USC cell lines when compared with normal endometrial cells $(P<0.05)$. RT-PCR and FACS analyses demonstrated that anti-mCRP siRNAs were effective in reducing CD46, CD55 and CD59 expression on USC $(P<0.05)$. Baseline complement-dependent cytotoxicity (CDC) against USC cell lines was low (mean \pm s.e.m. $=6.8 \pm 0.9 \%)$ but significantly increased upon CD55 and CD59 knockdown ( $11.6 \pm 0.8 \%$ and $10.7 \pm 0.9 \%$, respectively, $P<0.05$ ). Importantly, in the absence of complement, both CD55 and CD59, but not CD46, knockdowns significantly augmented ADCC against USC overexpressing Her2/neu.

CONCLUSION: Uterine serous carcinoma express high levels of the mCRPs CD46, CD55 and CD59. Small interfering RNA inhibition of CD55 and CD59, but not CD46, sensitises USC to both CDC and ADCC in vitro, and if specifically targeted to tumour cells, may significantly increase trastuzumab-mediated therapeutic effect in vivo.

British Journal of Cancer (2012) 106, 1543- 1550. doi:I0.1038/bjc.2012.132 www.bjcancer.com

(c) 2012 Cancer Research UK

Keywords: uterine serous cancer; Her2/neu; CD46; CD55; CD59; trastuzumab

Endometrial cancer (EC) is the most common gynaecological malignancy in women, with an incidence of 46470 new cases and 8120 deaths in 2011 in the United States (Siegel et al, 2011). Endometrial cancer is classified based on the clinical picture and histopathological pattern into Type I and Type II disease (Bohkman, 1983; Mutch, 2012). Type I disease includes grade-1 and -2 tumours with endometrioid histology, is oestrogendependent and usually preceded by endometrial hyperplasia. This cancer typically occurs in obese patients and is associated with diabetes and hypertension. Most patients are typically diagnosed at an early stage secondary to postmenopausal bleeding and have a good prognosis (Bohkman, 1983; Rose, 1996). In contrast, Type II

*Correspondence: Dr AD Santin; E-mail:-alessandro.santin@yale.edu Received 9 February 2012; revised 9 March 2012; accepted 14 March 2012
EC, which includes uterine serous carcinoma (USC), clear cell cancer and grade-3 endometrioid carcinoma, typically occurs in older thinner patients and is not hormone-dependent (Bohkman, 1983; Mutch, 2012). These tumours are more aggressive and have a worse prognosis than Type I EC (Rose, 1996).

Uterine serous carcinoma represents the most aggressive subtype of EC (Hendrickson et al, 1982). Uterine serous carcinoma has been associated with women of African-American ethnicity, tamoxifen use and BRCA gene mutations and is characterised by a high propensity for early lymphovascular invasion, as well as intraperitoneal and extra-abdominal spread at the time of presentation (Hendrickson et al, 1982; El-Sahwi et al, 2012). The overall 5-year survival is about $30 \%$ for all stages and the recurrence rate after surgery is extremely high (50-80\%) (Schwartz, 2006; El-Sahwi et al, 2012). Thus, there is a dire need for the development of novel, target-specific and more effective therapeutic strategies against this rare subset of EC. 
The $c$-erbB2 gene encodes for erbB2 (Her2/neu), a member of the erbB-receptor tyrosine kinase family. This is a family of four transmembrane glycoproteins (erbB1, erbB2, erbB3 and erbB4) that are expressed on epithelial, mesenchymal and neuronal cells. Ligand-binding results in dimerisation of the receptor either with a twin receptor (homodimerisation) or with one of its siblings (heterodimerisation) (Yarden and Sliwkowski, 2001). This leads to phosphorylation of intracellular tyrosine kinase residues that serve as docking sites for effectors and transcription factors that ultimately modulate a variety of biological responses, such as proliferation, survival, migration and differentiation. Our group and others, including the Gynaecologic Oncology Group in cooperative multicenter studies, have reported Her2/neu overexpression (i.e., $2+$ and/or $3+$ by immunohistochemistry (IHC) in $40-60 \%$ of patients harbouring USC (Santin et al, 2005a, b, c; Díaz-Montes et al, 2006; Morrison et al, 2006; Grushko et al, 2008). Furthermore, in previous reports, patients with USC overexpressing the Her2/neu receptor have been found to have a worse prognosis than those who do not (Santin et al, 2005a, c; Morrison et al, 2006). These findings have provided a rationale to the use of Her2/neu-targeted therapies in patients harbouring this aggressive EC subtype.

Trastuzumab (Herceptin; Genentech, San Francisco, CA, USA) is a humanised monoclonal antibody $(\mathrm{mAb})$ directed against the Her2/neu receptor. This $\mathrm{mAb}$ has been shown to be highly effective in patients harbouring breast cancer overexpressing Her2/neu (Slamon et al, 2001; Baselga et al, 2005). Of interest, randomized phase II studies are currently evaluating the potential activity of trastuzumab in combination with chemotherapy in USC patients with advanced/recurrent disease (clinicaltrials.gov/ct2/show/ NCT01367002).

Efficacy of cancer immunotherapy with antibodies activating cellular and complement-mediated cytotoxicity is known to be potentially limited by the overexpression of multiple membranebound complement-regulatory proteins (mCRPs: CD46, CD55 and CD59) on the surface of tumour cells (Gelderman et al, 2004, 2005). However, to our knowledge, no study has yet investigated whether biologically aggressive USC may overexpress mCRPs and whether downregulation of CD46, CD55 and CD59 by small interfering RNA (siRNA) may sensitise USC overexpressing HER2/neu to complement and/or trastuzumab-dependent cell-mediated cytotoxicity. To fill this gap in knowledge, in this study we analysed 15 primary USC cell lines for CD46, CD55, CD59, Her2/neu receptor expression and $c$-erbB2 gene amplification and investigated the ability of siRNA against these mCRPs to sensitise USC to complement and antibody (trastuzumab)-induced cellular cytotoxicity in vitro.

\section{MATERIALS AND METHODS}

\section{Establishment of USC cell lines}

A total of 15 primary USC cell lines (USPC-ARK-1-USPC-ARK-15) were established after sterile processing of tumour samples from surgical biopsy specimens, as described previously (El-Sahwi et al, 2010), under approval of the Institutional Review Board. Tumours were staged according to the International Federation of Gynecologists and Obstetricians 1988 operative staging system. Source-patient characteristics of these 15 USC cell lines are described in Table 1.

\section{Her2/neu immunostaining of formalin-fixed tumour tissues}

Formalin-fixed, paraffin-embedded tissue blocks from the USC patients from whom primary cell lines were established were retrieved from surgical pathology files. Specimens were reviewed by a gynaecologic pathologist. The level of Her2/neu expression was evaluated on the most representative block by the standard immunohistochemical staining, using the Hercept test (Dako, Glostrup, Denmark), as previously described (El-Sahwi et al, 2010).

\section{Fluorescent in-situ hybridisation (FISH)}

Fluorescent in-situ hybridisation analysis was performed on either cell blocks or formalin-fixed paraffin-embedded tissue blocks from all USCs using the PathVysion Her-2 DNA FISH Kit (Abbott Molecular Inc., Abbott Park, IL, USA) according to the manufacturer's instructions, as previously described (El-Sahwi et al, 2010).

\section{Quantitative real-time PCR (qRT-PCR)}

RNA isolation from all the 15 primary USC cell lines was performed using TRIzol Reagent (Invitrogen, Carlsbad, CA, USA), according to the manufacturer's instructions. Quantitative PCR was carried out with a 7500 RealTime PCR System using the manufacturer's recommended protocol (Applied Biosystems, Foster City, CA, USA) to evaluate the expression of CD46, CD55 and CD59 mCRPs in all samples. The primers and probe for CD46, CD55 and CD59 were obtained from Applied Biosystems (i.e., CD46 Assay ID: Hs00611257_m1, CD55 Assay ID: Hs00892618_m1 and CD59 Assay ID: Hs00174141_m1). The comparative threshold cycle method was used to determine gene expression in each sample, relative to the value observed in non-malignant endometrial epithelial cell samples collected from similar-age women, using glyceraldehyde-3-phosphate dehydrogenase (Assay ID Hs99999905_m1) RNA as an internal control.

\section{Small interfering RNA knockdown experiments}

CD46-, CD55- and CD59-specific siRNA oligonucleotides (i.e., CD46: 5'-GGAUACUUCUAUAUACCUCUU- $3^{\prime}$ (sense) and $3^{\prime}$-UUCCUAUGAAGAUAUAUGGAG- $5^{\prime}$ (antisense); CD55:5'-AUGU GAAGAAAGCUUUGUGUU- $3^{\prime}$ (sense) and $3^{\prime}$-UUUACACUUCUU UCGAAACAC-5' (antisense); CD59: 5'-GGACCUGUGUAACUUU AACUU- $3^{\prime}$ (sense) and $3^{\prime}$-UUCCUGGACACAUUGAAAUUG- ${ }^{\prime}$ (antisense)) and nonspecific siRNA duplexes used as negative controls, were purchased from Ambion, Inc. (Austin, TX, USA). Briefly, representative USC cell lines harbouring amplification of the $c$-erbB2 gene were cultured in six-well plates and transfected with anti-CD46, anti-CD55 or anti-CD59 siRNA duplexes at $10 \mathrm{~nm}$ in conjunction with $5 \mu$ l Lipofectamine RNAiMAX (Invitrogen) following the manufacturer's instructions. Mock transfections and nonspecific siRNA duplexes were used as negative controls. USC cells were treated for $72 \mathrm{~h}$ (i.e., the time we found required for maximal downregulation of CD46, CD55 or CD59, based on flow cytometry and qRT-PCR), after which they were used in CDC and antibody (trastuzumab)-dependent cellular cytotoxicity (ADCC) as described below.

\section{Flow cytometry}

The clinically marketed anti-Her2/neu trastuzumab (Herceptin; Genentech), a humanised mAb of the G1 type that binds with high affinity to the extracellular domain of the HER2/neu receptor and commercially available antibodies against mCRPs CD46 (cat. no. 555948), CD55 (cat. no. 555691) and CD59 (cat. no. 555761) (BD Pharmingen, San Diego, CA, USA), were used for our study. Primary USC cell lines obtained from the above described patients were stained with $2.5 \mu \mathrm{g} \mathrm{ml}^{-1}$ of trastuzumab or $5 \mu \mathrm{g}$ per $200 \mu \mathrm{l}$ of anti-CD46, anti-CD55 or anti-CD59 for $30 \mathrm{~min}$ on ice. A total of $5 \mu \mathrm{g} \mathrm{ml}^{-1}$ of the chimeric anti-CD20 mAb rituximab (Rituxan; Genentech) was used as a negative control. Fluorescein isothiocyanate-conjugated goat antihuman $\mathrm{F}(\mathrm{ab} 1) 2$ immunoglobulin (BioSource International) or goat-anti-mouse FITC (BD Pharmingen) were used for staining as a secondary reagents. 
Table I Patient characteristics from which the 15 USC cell lines were established

\begin{tabular}{|c|c|c|c|c|c|c|}
\hline Patient & Age & Race & Stage & Histology & IHC Her2/neu & FISH Her $2 /$ neu \\
\hline USPC ARK-I & 62 & AA & IVA & Pure & $3+$ & Positive \\
\hline USPC ARK-2 & 63 & AA & IVB & Pure & $3+$ & Positive \\
\hline USPC ARK-3 & 59 & $\mathrm{AA}$ & IVB & Mixed & $3+$ & Positive \\
\hline USPC ARK-4 & 73 & C & IVB & Pure & $1+$ & Negative \\
\hline USPC ARK-5 & 73 & $\mathrm{AA}$ & IIIC & Pure & $1+$ & Negative \\
\hline USPC ARK-6 & 62 & C & IB & Mixed & $1+$ & Negative \\
\hline USPC ARK-7 & 75 & C & $\| C$ & Pure & $2+$ & Negative \\
\hline USPC ARK-8 & 88 & C & IIIA & Pure & $1+$ & Negative \\
\hline USPC ARK-9 & 73 & AA & $\| \mathrm{IIC}$ & Mixed & $3+$ & Positive \\
\hline USPC ARK-IO & 79 & C & IVB & Pure & $3+$ & Positive \\
\hline USPC ARK-II & 80 & $\mathrm{AA}$ & IIIC & Mixed & $1+$ & Negative \\
\hline USPC ARK-I2 & 64 & C & IVB & Pure & 0 & Negative \\
\hline USPC ARK-I3 & 67 & C & IVB & Mixed & $2+$ & Negative \\
\hline USPC ARK-I4 & 73 & $A A$ & IV & Pure & 0 & Negative \\
\hline USPC ARK-I 5 & 67 & C & $\| \mathrm{IIC}$ & Pure & $1+$ & Negative \\
\hline
\end{tabular}

Abbreviations: $A A=$ African American; $C=$ Caucasian; USPC $=$ uterine serous papillary adenocarcinoma.

Analysis was conducted with a FACScalibur, using Cell Quest software (BD Biosciences).

\section{Tests for ADCC}

A standard 4 -h chromium $\left({ }^{51} \mathrm{Cr}\right)$-release assay was performed to measure the cytotoxic reactivity of Ficoll-PaqueTM PLUS-separated (GE Healthcare, Uppsala, Sweden) peripheral blood lymphocytes (PBLs) obtained from several healthy donors against USPC cell lines harbouring amplification of the c-erbB2 gene before and after knockdown in CD46, CD55 and CD59 expression by siRNA. The release of ${ }^{51} \mathrm{Cr}$ from the target cells was measured as evidence of tumour cell lysis after exposure of tumour cells to $5 \mu \mathrm{g} \mathrm{ml}^{-1}$ of trastuzumab. Additional controls included the incubation of target cells alone or with $\mathrm{PBL}$ or mAb separately. The chimeric anti-CD20 mAb rituximab was used as a negative control for trastuzumab in the bioassays. antibody (trastuzumab)dependent cellular cytotoxicity was calculated as percentage killing of target cells obtained after trastuzumab incubation with effector cells compared with ${ }^{51} \mathrm{Cr}$ release from target cells incubated alone.

\section{Complement-mediated target cell lysis of USC cell lines}

A standard $4-\mathrm{h}{ }^{51} \mathrm{Cr}$-release assay identical to the one used for ADCC assays was used, except that human plasma in a dilution of $1: 2$ was added in place of the effector cells in AIM-V culture medium (Invitrogen). This human plasma was used as a source of complement to test for complement-mediated target cell lysis before and after knockdown in CD46, CD55 and CD59 expression by siRNA in the presence of trastuzumab. In additional experiments, heat-inactivated $\left(56^{\circ} \mathrm{C}\right.$ for $\left.60 \mathrm{~min}\right)$ human plasma was diluted $1: 2$ before addition to the assay. Rituximab was used as isotype control mAb. In all experiments, controls included the incubation of target cells alone or with mAb separately.

\section{Statistical analysis}

For qRT-PCR data, the right-skewing was removed by taking copy number ratios relative to the lowest-expressing normal human EC sample ('relative copy number'), $\log _{2}$ transforming them to $\Delta \mathrm{C}_{\mathrm{t}} \mathrm{s}$ and comparing the results through unequal variance $t$-test for FISH-positive vs FISH-negative USC. Group means with 95\% confidence intervals (CIs) were calculated by computing them on the $\Delta \mathrm{C}_{\mathrm{t}} \mathrm{s}$ and then reverse-transforming the results to obtain means ( $95 \%$ CIs) of relative copy numbers. The analyses of differences in mCRP expression levels after knockdown of CD46, CD55 and CD59 by siRNA measured by RT-PCR and flow cytometry were performed using the Wilcoxon Mann-Whitney test. Kruskal-Wallis test and $\chi^{2}$ analysis were used to evaluate differences in CDC and trastuzumab-mediated ADCC levels in primary tumour cell lines before and after knockdown of CD46, CD55 and CD59 by siRNA. Statistical analysis was performed using PASW version 18 (SPSS, Chicago, IL, USA) and GraphPad Prism (GraphPad Software, Inc., LaJolla, CA, USA). A $P$-value of $<0.05$ was considered statistically significant.

\section{RESULTS}

\section{Her2/neu expression by IHC on USC}

Immunohistochemistry detecting Her2/neu expression was performed on formalin-fixed paraffin-blocks of USC tissues from which the 15 primary cell lines were established. As reported in Table 1,5 out of 15 specimens showed strong staining $(3+)$ for Her2/neu protein, whereas the remaining 10 showed weak to moderate staining $(0,1+$ and $2+)$.

\section{Fluorescent in-situ hybridisation}

Fluorescent in-situ hybridisation analysis was performed on either cell blocks or formalin-fixed paraffin-embedded tissue blocks from all USCs used in this study. c-erbB2 gene amplification was detected in all five primary USC specimens showing $3+$ positive expression by IHC (Table 1), suggesting that strong receptor expression by IHC and high Her2/neu mRNA level of these tumours (see below) is likely caused by gene amplification. In contrast, the remaining 10 USC cell lines were found to be negative for c-erbB2 gene amplification (Table 1).

\section{Flow cytometry}

Surface Her2/neu expression was evaluated by FACS analysis on all the 15 primary USC cell lines using trastuzumab. In addition, as negative controls, several B cell lines (EBV-transformed lymphoblastoid B cell lines) established from the same USC patients from which the tumour cell lines had been established were also studied (data not shown). In all, 4 out of 15 USC cell lines (all FISH positive) showed a very high expression of Her2/neu (mean fluorescence intensity (MFI) ranging from 228 to 339), whereas the remaining 11 ( $1 \mathrm{FISH}$ positive and $10 \mathrm{FISH}$ negative) were found to express significantly lower levels of Her2/neu (MFI ranging from 10 to 72 ) (Table 2, $P>0.01$ ). 
Table 2 Complement regulatory protein and Her2/neu expression by flow cytometry in uterine serous tumours

\begin{tabular}{|c|c|c|c|c|c|c|c|c|}
\hline \multirow[b]{2}{*}{ Cell lines } & \multicolumn{2}{|c|}{ CD46 } & \multicolumn{2}{|c|}{ CD55 } & \multicolumn{2}{|c|}{ CD59 } & \multicolumn{2}{|c|}{ Her2/neu } \\
\hline & $\%$ & MFI & $\%$ & MFI & $\%$ & MFI & $\%$ & MFI \\
\hline USPC-ARK-I & 99 & $54^{\mathrm{a}}$ & 98 & 43 & 100 & 102 & 100 & 339 \\
\hline USPC-ARK-2 & 99 & 64 & 100 & 59 & 99 & 45 & 100 & 230 \\
\hline USPC-ARK-3 & 100 & 64 & 100 & 71 & 100 & 55 & 100 & 228 \\
\hline USPC-ARK-4 & 100 & 51 & 100 & 73 & 100 & 160 & 95 & 10 \\
\hline USPC-ARK-5 & 98 & 33 & 98 & 52 & 95 & 32 & 84 & 18 \\
\hline USPC-ARK-6 & 100 & 70 & 98 & 37 & 98 & 39 & 93 & 13 \\
\hline USPC-ARK-7 & 100 & 24 & 53 & 20 & 100 & 46 & 95 & 47 \\
\hline USPC-ARK-8 & 100 & 115 & 100 & 97 & 100 & 84 & 83 & 24 \\
\hline USPC-ARK-9 & 99 & 123 & 99 & 107 & 99 & 85 & 99 & 285 \\
\hline USPC-ARK-I0 & 100 & 54 & 100 & 46 & 100 & 57 & 99 & 25 \\
\hline USPC-ARK-II & 100 & 163 & 99 & 67 & 99 & 123 & 85 & 23 \\
\hline USPC-ARK-I2 & 99 & 49 & 100 & 59 & 58 & 19 & 99 & 32 \\
\hline USPC-ARK-I3 & 95 & 48 & 91 & 35 & 64 & 22 & 100 & | | \\
\hline USPC-ARK-I 4 & 99 & 60 & 100 & 61 & 85 & 31 & 99 & 26 \\
\hline USPC-ARK-I 5 & 99 & 45 & 99 & 50 & 57 & 20 & 100 & 72 \\
\hline
\end{tabular}

Abbreviations: $\mathrm{MFI}=$ mean fluorescence intensity; $\mathrm{USPC}=$ uterine serous papillary

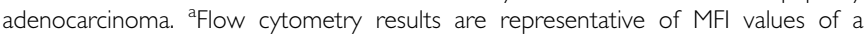
minimum of three independent experiments for USC cell line.

\section{qRT-PCR}

A total of 15 primary USC cell lines were tested by RT-PCR for the expression of CD46, CD55 and CD59 mCRP at mRNA level. Expression for CD46, CD55 and CD59 mCRP was detected in all 15 USC cell lines tested. CD46, CD55 and CD59 mRNA transcripts were $17.6 \pm 3.8,3.0 \pm 1.0$ and $7.2 \pm 1.3$ fold higher, respectively, (mean \pm s.e.) when compared with the level of expression found in normal control endometrial cells $(P<0.05)$. No significant differences in mCRP expression for CD46, CD55 and CD59 were detected when USC cell lines with high HER2/neu expression (i.e., FISH positive) were compared with those with low HER2/neu expression (i.e., FISH negative, data not shown).

\section{mCRP expression by flow cytometry}

To determine whether the expression of CD46, CD55 and CD59 mCRP mRNA detected using qRT-PCR in USC cell lines also resulted in expression of the protein on the surface of tumour cells, flow cytometry was performed on all primary cell lines. Membrane-bound complement-regulatory proteins surfaceexpression results from flow-cytometry analysis were found to be in good agreement with mCRP expression results found by qRTPCR in primary cell lines (Table 2). No significant differences were found between high $v s$ low HER2/neu expressor USC cell lines for any of the mCRP tested (Table 2 and data not shown).

\section{Downregulation of $\mathrm{mCRP}$ expression by anti-CD46, anti-CD55 and anti-CD59 siRNA}

Uterine serous carcinoma cell lines harbouring amplification of c-erbB2 by FISH were transfected with selected siRNA specific for CD46, CD55 and CD59 and inhibition of individual mCRP knockdown was evaluated by FACS analysis and RT-PCR at different time points. We found the best inhibition rates for antiCD46, anti-CD55 and anti-CD59 siRNA at $72 \mathrm{~h}$ after transfection (data not shown). Upon optimisation, CD46 protein expression was decreased by siRNA by $83 \%$ in USPC-ARK-2 (Figure 1 ) and by $71 \%$ in USPC-ARK-3 (data not shown). CD55 protein expression was decreased by siRNA by $51 \%$ in USPC-ARK- 2 and by $53 \%$ in USPC-ARK-3, whereas CD59 protein expression was decreased by
siRNA by $92 \%$ in USPC-ARK-2 and by $93 \%$ in USPC-ARK-3 $(P<0.05)$ (Figure 1). qRT-PCR analysis at $72 \mathrm{~h}$ after transfection with siRNA specific for CD46, CD55 and CD59 confirmed the results obtained at protein levels by flow cytometry. Specifically, we found a mean downregulation of CD46 expression by siRNA by $86 \%$ in USPC-ARK-2 and by $93 \%$ in USPC-ARK-3. CD55 expression was decreased by siRNA by $77 \%$ in USPC-ARK- 2 and by $78 \%$ in USPC-ARK-3, whereas CD59 expression was decreased by siRNA by $97 \%$ in USPC-ARK- 2 and by $98 \%$ in USPC-ARK-3 $(P<0.05)$

\section{Effects of knockdown of CD46, CD55 and CD59 by siRNA on CDC in USC cell lines overexpressing HER2/neu}

In order to evaluate the effect of complement against USC cell lines, human plasma diluted $1: 2$ (with and without heat inactivation) was added during the standard $4-\mathrm{h}{ }^{51} \mathrm{Cr}$-release cytotoxicity assays against USPC-ARK-2 and USPC-ARK-3 tumour cell lines expressing high levels of Her2/neu before and after knockdown of CD46, CD55 and CD59 by siRNA. As representatively shown in Figure 2, for both USC cell lines, in the absence of PBL the addition of untreated plasma in the presence of trastuzumab induced low levels of cytotoxicity against the USC cell lines (mean \pm s.e.m. $=6.8 \% \pm 0.9 \%$ ). Importantly, downregulation of CD55 and CD59, but not CD46, significantly increased CDC against both tumours. Mean killing \pm s.e.m. after knockdown of CD55 and CD59 was $11.6 \pm 0.8 \%$ and $10.7 \pm 0.9 \%$, respectively, (Figure $2, P<0.05$ ). Inactivation of human plasma by heat reduced $\mathrm{CDC}$ to negligible levels (i.e., $<1 \%$, data not shown). These results demonstrating the importance of CD55 and CD59 expression in potentially inhibiting complement attack against USC cell lines overexpressing Her2/neu in the presence of trastuzumab. In additional experiments, we evaluated the effect of the simultaneous block of all three mCRP by anti-CD46, anti-CD55 and anti-CD59 siRNA against USPC-ARK-2 and USPC-ARK-3 tumour cell lines in CDC experiments. Mean killing \pm s.e.m. after the simultaneous knockdown of CD46, CD55 and CD59 was $13.6 \pm 1.8 \%$ and $11.9 \pm 1.1 \%$ in USPC-ARK-2 and USPC-ARK-3, respectively $(P<0.05)$.

\section{Effects of knockdown of CD46, CD55 and CD59 by siRNA} on ADCC in USC cell lines overexpressing HER2/neu

Two representative primary USC cell lines (i.e., USPC-ARK-2 and USPC-ARK-3) harbouring amplification of the c-erbB2 were tested for their sensitivity to natural killer (NK) cytotoxicity when challenged with heterologous PBL, collected from several healthy donors, in a standard $4-\mathrm{hr}{ }^{51} \mathrm{Cr}$-release assay before and after knockdown of CD46, CD55 and CD59 by siRNA in the absence of plasma as source of complement. Both cell lines were found to be highly resistant to NK-mediated cytotoxicity when combined with PBLs at effector to target cell ratios (E:T) varying from $10: 1$ to 20:1 in the absence of trastuzumab (mean killing \pm s.e.m. $=$ $3.0 \% \pm 2.1$ with all E:T). Similarly, both USC cell lines incubated with rituximab control antibody and PBL were not significantly killed (mean killing \pm s.e.m. $=2.9 \pm 2.0 \%$ with all $\mathrm{E}: \mathrm{T}$, data not shown). In contrast, as demonstrated in Figure 3, USPC-ARK-2 and USPC-ARK-3 were highly sensitive to trastuzumab-mediated ADCC (mean killing \pm s.e.m. $=48.0 \pm 1.1 \%$ at $20: 1$ ratio). We then evaluated the sensitivity of USPC-ARK-2 and USPC-ARK-3 cell lines to NK cytotoxicity and trastuzumab after knockdown of CD46, CD55 and CD59 by siRNA. We found downregulation of CD55 and CD59 to consistently and significantly increase NKmediated killing in both the cell lines in multiple experiments (mean killing \pm s.e.m. $=62.5 \pm 2.1 \%$ and $64.8 \pm 2.1 \%$ at $20: 1$ ratio, respectively, $P<0.05$, Figure 3 ). In contrast, downregulation of CD46 by siRNA did not significantly alter NK-mediated killing in 

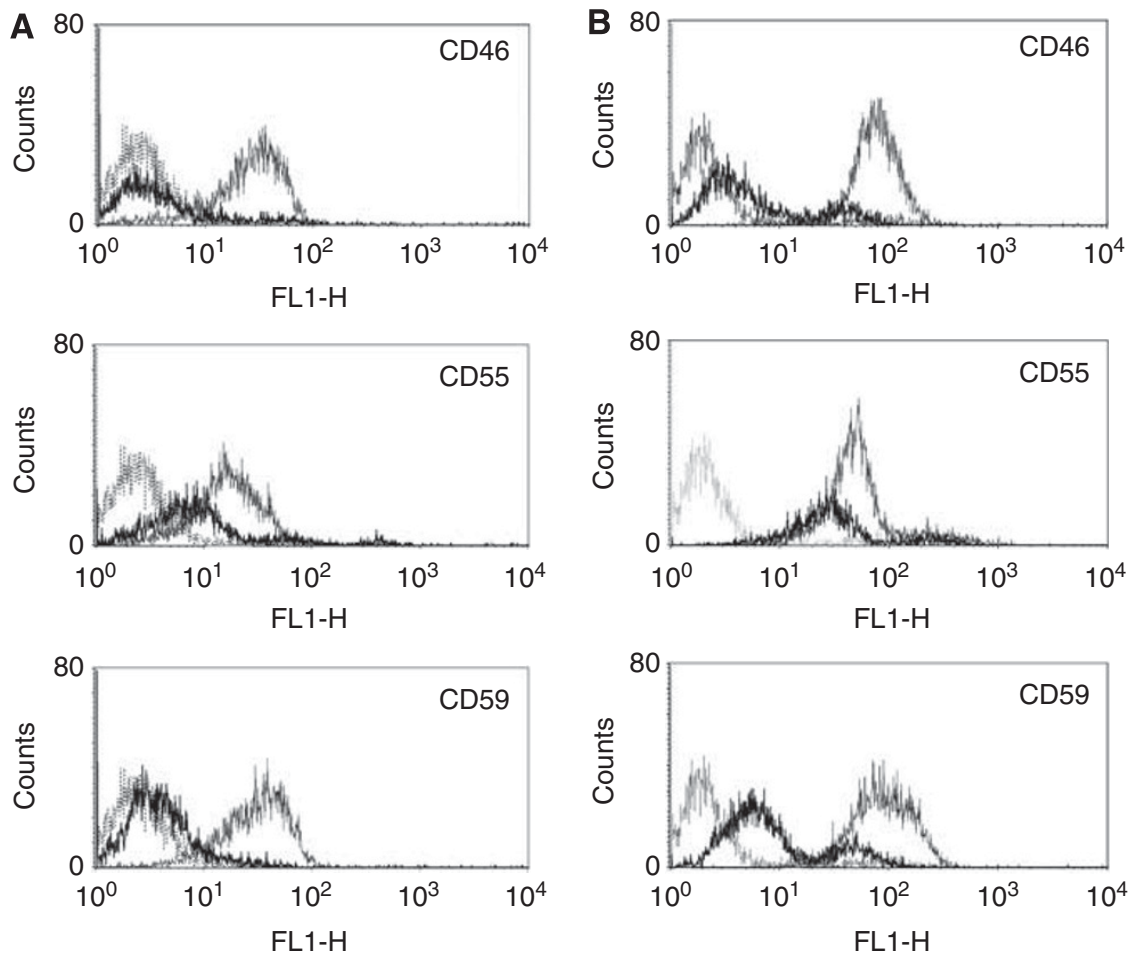

Figure I (A) Representative FACS histograms showing knockdown of the mCRPs CD46, CD55 and CD59 in uterine serous papillary carcinoma cell line USPC-ARK-2. (B) Representative FACS histograms showing knockdown of the mCRPs CD46, CD55 and CD59 in uterine serous papillary carcinoma cell line USPC-ARK-3. The faint line depicts CD46, CD55 and CD59 expression in USPC cell lines before transfection with each mCRP-specific siRNA. The bold line describes CD46, CD55 and CD59 expression after transfection with each mCRP-specific siRNA. Dotted line: isotype control. A significant downregulation of mCRP expression by anti-CD46, anti-CD55 and anti-CD59 siRNA was noted in both cell lines tested $(P<0.05)$.

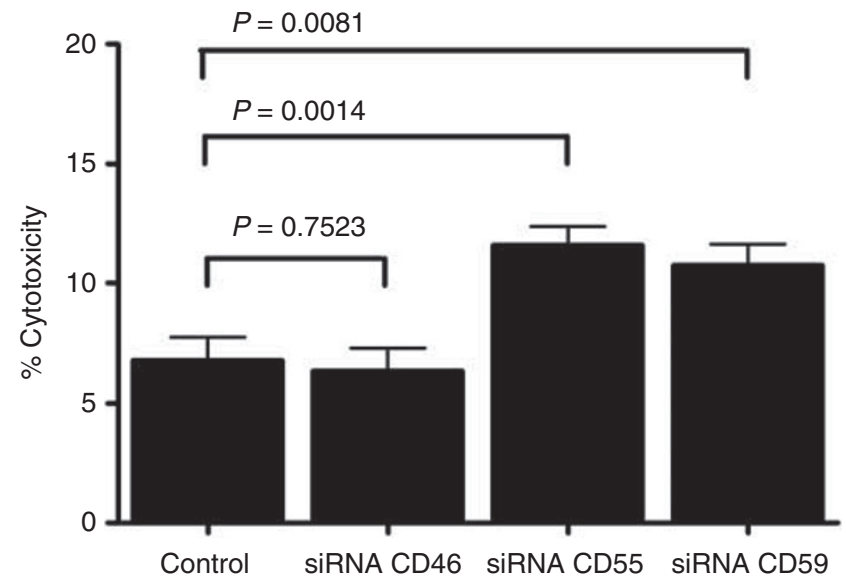

Figure 2 Pooled cytotoxicity data in the setting of siRNA against CD46, CD55 and CD59 in uterine serous papillary carcinoma. Silencing of CD55 and CD59, but not CD46, augments CDC. Mean \pm s.e.m. are shown representing 10 replicates using two cell lines. Controls in the experiments included both mock-transfected and USC cells pretreated with nonspecific siRNA as described in the methods section.

either cell line (mean killing \pm s.e.m. $=45.9 \pm 0.7 \%$ at $20: 1$ ratio, Figure 3).

\section{DISCUSSION}

Resistance of tumour cells to lysis mediated by NK cells and homologous complement by upregulation of mCRP, such as

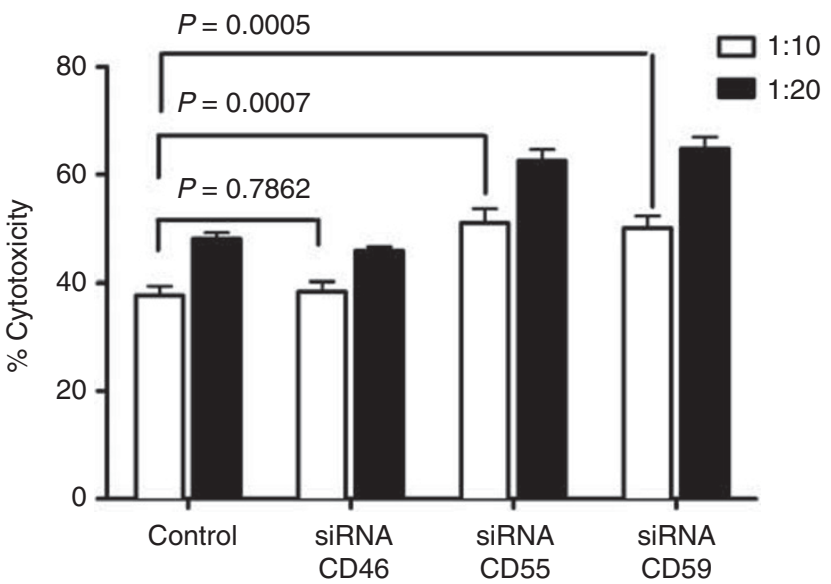

Figure 3 Pooled antibody-dependent cell-mediated cytotoxicity at effector to target ratios of $10: 1$ and $20:$ I in the setting of siRNA against CD46, CD55 and CD59 in uterine serous papillary carcinoma. Silencing of CD55 and CD59, but not CD46, enhances trastuzumab-dependent cellmediated cytotoxicity at all concentrations examined. Mean \pm s.e.m. are shown representing 18 replicates across two cell lines. P-values for 10: I ratios: are provided; corresponding $P$-values for control vs siRNA CD46, siRNA CD55 and siRNA CD59 at 20: I are 0.1225, <0.000I, <0.000I, respectively.

membrane cofactor protein (CD46), decay-accelerating factor (CD55) and protectin (CD59), are potentially major immuneescape strategies developed by tumours (reviewed in Gelderman et al, 2004, 2005). Indeed, in many tumours, mCRP expression is 
greater than in normal surrounding tissues and mCRP act as physiological brakes to complement amplification on normal as well as tumour cells either by limiting formation of the C3/C5 convertase enzymes (i.e., CD46 and CD55) or by assembly of the cytolytic membrane-attack complex (MAC, i.e., CD59) (reviewed in Gelderman et al, 2004, 2005). Consistent with this view, cells from patients with the haematological disorder paroxysmal nocturnal hemoglobinuria are defective in the machinery for glycosylphosphatidylinisotol-anchor synthesis and hence lack surface expression of both CD55 and CD59 and are highly susceptible to the lytic effect of MAC (Iida et al, 1994). Importantly, in vitro blocking studies of CD55 and CD59 by antibodies or miniantibodies have been reported to increase not only complement-mediated lysis but also antibody-dependent cellmediated cytotoxicity against multiple human and rat tumours in the absence of complement (Finberg et al, 1992; Caragine et al, 2002; Macor et al, 2007). These findings suggesting additional noncomplement-dependent function for these mCRP are noteworthy because, although multiple mechanisms of action have been attributed to clinically approved antibodies targeting specific targets on human tumours such as trastuzumab (i.e., anti-HER2/ neu), rituximab (i.e., anti-CD20) or cetuximab (i.e., anti-EGFr), including inhibition of tumour proliferation, angiogenesis and metastasis, induction of apoptosis and/or promotion of cell cycle arrest, strong experimental evidence currently suggests that engagement of Fc receptors on effector cells (i.e., mainly NK cells) represents the dominant component of the in vivo activity of these mAbs against human tumours (Clynes et al, 2000). In this regard, NK cells are considered the best-suited lymphocytes for ADCC because they carry Fc $\gamma$ RIII receptors, which are activator molecules, and not Fc $\gamma$ RIIb receptors, which inhibit ADCC (Clynes et al, 2000). In support of this hypothesis, the efficacy of the anti-EGFR antibody (M225) when used as an F(ab)2 in blocking the growth of tumours in vivo has been previously demonstrated to be only $50 \%$ of the activity shown by the intact antibody when it is able to engage the $\mathrm{Fc}$ receptors on NK cells (Fan et al, 1993). Similarly, the engagement of Fc $\gamma$ RIII receptors on NK effectors cells has been demonstrated to represent the dominant component of the in vivo activities of trastuzumab and rituximab in multiple animal models (Clynes et al, 2000). On the basis of this evidence, it seems therefore likely that specific inhibition of mCRP, such as CD46, CD55 and CD59, on USC harbouring amplification of the c-erbB2 gene may have the potential to sensitise further these biologically aggressive tumours to trastuzumab-mediated CDC and ADCC.

In the current study, using multiple techniques, we demonstrated high Her2/neu expression in 5 out of 15 primary USC cell lines established in our laboratory. We also confirmed that USC cell lines overexpressing Her2/neu are sensitive to NK-mediated cytotoxicity in the presence of trastuzumab (El-Sahwi et al, 2010). Importantly, because of the lack of information regarding expression of mCRP on USC, we carefully investigated CD46, CD55 and CD59 expression in all the 15 primary USC cell lines and analysed whether siRNA against CD46, CD55 or CD59 may sensitise USC to CDC and ADCC in vitro. We report for the first time increased expression of mCRP in all primary USC available to us (i.e., 15 primary USC cell lines) regardless of their high or low HER2/neu expression. These results are consistent with the upregulation of CD46, CD55 or CD59 mCRP previously reported in various human tumours, including colorectal cancer, cervical cancer, prostate cancer and renal cell carcinoma (reviewed in Gelderman et al, 2004, 2005). Although the mechanisms related to activation/upregulation of these mCRP in USC are currently poorly understood, it is of interest that CD59 activation has been previously reported to be a result of increased acetylation of p53 (Donev et al, 2006), an oncosuppressor gene known to represent one of the most commonly overexpressed/mutated gene in USC (Kohler et al, 1992). Whatever the mechanism, the common upregulation of mCRP in USC suggests that high expression of CD46, CD55 or CD59 may represent an additional molecular evasion strategy for these biologically aggressive endometrial tumours during the interaction with the host immune system.

Consistent with this hypothesis, in an attempt to understand the implication of downregulating CD46, CD55 and CD59 in USC overexpressing Her2/neu during trastuzumab-induced cytotoxicity, in this study, we have compared trastuzumab CDC in the presence or absence of siRNA specifically targeting each of these mCRP in bioassays. Although the baseline level of CDC with or without trastuzumab was low against USC cell lines, a significant increase in trastuzumab-mediated CDC was consistently detected after downregulation of CD55 and CD59 by siRNA in USC cell lines. In contrast, no significant increase in CDC was detected after downregulation of CD46 by siRNA in any of the USC cell lines studied. These data are in agreement with a number of previous studies showing an innate high resistance of USC to complement actvity in vivo and in vitro (El-Sahwi et al, 2010), as well as previous reports showing that in classical pathway activation, CD55 and CD59, but not CD46, are most effective in preventing cell lysis (Gorter and Meri, 1999). Importantly, our current findings provide for the first time evidence that the high resistance to complement of these biologically aggressive endometrial tumours is at least in part related to overexpression of CD55 and CD59.

Next, primary USC cell lines overexpressing Her2/neu were tested in trastuzumab ADCC experiments in the presence of siRNA against CD46, CD55 and CD59 and PBL as source on NK cells but in the absence of complement. We found a significant increase in trastuzumab-mediated ADCC after downregulation of CD55 and CD59 by siRNA in USC cell lines. In contrast, no significant effect was noted after downregulation of CD46 by siRNA. Although surprising, these results obtained in the absence of serum as source of complement are in agreement with those of other groups who have previously suggested additional novel functions for CD55 and CD59 in tumour immune surveillance that may be unrelated to complement inhibition (Finberg et al, 1992; Caragine et al, 2002; Kusama et al, 2003; Macor et al, 2007). Indeed, blocking of CD55 and CD59 by antibodies and/or miniantibodies was recently reported to increase not only complement but also PBL-mediated killing of human lymphoma cells triggered by rituximab (Macor et al, 2007). Similarly, Finberg et al (1992) found that in the absence of serum, CD55 expression on target cells significantly inhibited cytotoxicity by human NK cells. Although not specifically tested in our experiments, it is possible, as previously documented by others (Finberg et al, 1992), that in the absence of serum, NK cells used in the assays may release soluble C3 in the culture medium and thereby influence the cytolytic process. Whatever the mechanism, these data combined with our results suggest that the high levels of expression of CD55 and CD59 mCRP found in USC may not only decrease CDC but also, affecting trastuzumab ADCC, function as a negative regulator of cellular immunity in vitro and likely in vivo.

Her2/neu overexpression and c-erbB2 gene amplification are detected in a significant number of USC patients (Díaz-Montes et al, 2006; Grushko et al, 2008) and found to be associated with more aggressive biological behaviour and poor prognosis (Santin et al, 2005a, c; Morrison et al, 2006). These findings have led to consideration of Her2/neu as a potential marker for trastuzumabbased treatment in patients harbouring USC refractory to the standard adjuvant treatment (Villella et al, 2006; Santin et al, 2008). Consistent with this view, a prospective randomized clinical trial comparing the efficacy of trastuzumab in combination with carboplatin and paclitaxel $v s$ carboplatin and paclitaxel alone in advanced/recurrent USC patients overexpressing Her2/neu is currently recruiting patients in multiple institutions in the USA (clinicaltrials.gov/ct2/show/NCT01367002). Taken together, the results of our current study suggest that the simultaneous attack of HER2/neu-overexpressing USC with trastuzumab and with 
siRNA able to specifically target CD55 and CD59 in vivo might improve both $\mathrm{CDC}$ and trastuzumab ADCC against these biologically aggressive tumours. In this regard, although the lack of targeted siRNA delivery currently remains a major obstacle for its broad therapeutic in vivo application, recent evidence suggests that specific targeting of mCRP by nanoparticles and/or herceptinconjugated liposomes is feasible and potentially able to improve antibody-based cancer immunotherapy (Aigner, 2007; Li et al, 2007, 2009).

In conclusion, this is the first report demonstrating that biologically aggressive USC express high levels of the mCRPs CD46, CD55 and CD59 and that siRNA inhibition of CD55 and CD59 is able to sensitise USC to both CDC and ADCC in vitro. Although in vivo data will ultimately be necessary to validate the therapeutic potential of trastuzumab in combination with inhibition of CD55 and CD59, our in vitro results strongly suggest that

\section{REFERENCES}

Aigner A (2007) Applications of RNA interference: current state and prospects for siRNA-based strategies in vivo. Appl Microbiol Biotechnol 76: $9-21$

Baselga J, Carbonell X, Castañeda-Soto NJ, Clemens M, Green M, Harvey V, Morales S, Barton C, Ghahramani P (2005) Phase II study of efficacy, safety, and pharmacokinetics of trastuzumab monotherapy administered on a 3-weekly schedule. J Clin Oncol 23(10): 2162-2171

Bohkman JV (1983) Two pathogenetic types of endometrial carcinoma. Gynecol Oncol 15: 10-17

Caragine TA, Imai M, Frey AB, Tomlinson S (2002) Expression of rat complement control protein Crry on tumor cells inhibits rat natural killer cell-mediated cytotoxicity. Blood 100: 3304-3310

Clynes RA, Towers TL, Presta LG, Ravetch JV (2000) Inhibitory Fc receptors modulate in vivo cytoxicity against tumor targets. Nat Med 6: 443-446

Díaz-Montes TP, Ji H, Smith Sehdev AE, Zahurak ML, Kurman RJ, Armstrong DK, Bristow RE (2006) Clinical significance of Her-2/neu overexpression in uterine serous carcinoma. Gynecol Oncol 100: 139-144

Donev RM, Cole DS, Sivasankar B, Hughes TR, Morgan BP (2006) p53 regulates cellular resistance to complement lysis through enhanced expression of CD59. Cancer Res 66: 2451-2458

El-Sahwi K, Bellone S, Cocco E, Cargnelutti M, Casagrande F, Bellone M, Abu-Khalaf M, Buza N, Tavassoli FA, Hui P, Silasi DA, Azodi M, Schwartz PE, Rutherford TJ, Pecorelli S, Santin AD (2010) In vitro activity of pertuzumab in combination with trastuzumab in uterine serous papillary adenocarcinoma. Br I Cancer 102: 134-143

El-Sahwi K, Schwartz PE, Santin AD (2012) Development of targeted therapy in uterine serous carcinoma, a biologically aggressive variant of endometrial cancer. Expert Review of Anticancer Therapy 12(1): 41-49

Fan Z, Masui H, Altas I, Mendelsohn J (1993) Blockade of epidermal growth factor receptor function by bivalent and monovalent fragments of 225 anti-epidermal growth factor receptor monoclonal antibodies. Cancer Res 53: 4322-4328

Finberg RW, White W, Nicholson-Weller A (1992) Decay-accelerating factor expression on either effector or target cells inhibits cytotoxicity by human natural killer cells. Journal of Immunology 149(6): 2055-2060

Gelderman KA, Tomlinson S, Ross GD, Gorter A (2004) Complement function in mAb-mediated cancer immunotherapy. Trends Immunol 25: $158-164$

Gelderman KA, Lam S, Gorter A (2005) Inhibiting complement regulators in cancer immunotherapy with bispecific mAbs. Expert OpinBiol Ther 5: 1593-1601

Gorter A, Meri S (1999) Immune evasion of tumor cells using membrane bound complement proteins. Immunol Today 20: 576-582

Grushko TA, Filiaci VL, Mundt AJ, Ridderstråle K, Olopade OI, Fleming GF. Gynecologic Oncology Group (2008) An exploratory analysis of HER-2 amplification and overexpression in advanced endometrial carcinoma: a Gynecologic Oncology Group study. Gynecol Oncol 108: 3-9

Hendrickson M, Ross J, Eifel P, Martinez A, Kempson R (1982) Uterine papillary serous carcinoma: a highly malignant form of endometrial adenocarcinoma. Am J Surg Pathol 6: 93-108
siRNA inhibition of these mCRP, if specifically targeted to tumour cells, may represent an attractive and novel strategy to treat USC overexpressing HER2/neu refractory or resistant to chemotherapy.

\section{ACKNOWLEDGEMENTS}

Supported in part by grants from NIH R01 CA122728-01A4 and R01 CA154460-01A1, the Honorable Tina Brozman Foundation and Deborah Bunn Alley Ovarian Cancer Research Foundation to ADS. This investigation was also supported by the NIH Research Grant CA-16359 from the National Cancer Institute.

\section{Conflict of interest}

The authors declare no conflict of interest.
Iida Y, Takeda J, Miyata T, Inoue N, Nishimura J, Kitani T, Maeda K, Kinoshita T (1994) Characterization of genomic PIG-A gene: a gene for glycosylphosphatidylinositol-anchor biosynthesis and paroxysmal nocturnal hemoglobinuria. Blood 83(11): 3126-3131

Kohler MF, Berchuck A, Davidoff AM, Humphrey PA, Dodge RK, Iglehart JD, Soper JT, Clarke-Pearson DL, Bast Jr RC, Marks JR (1992) Overexpression and mutation of p53 in endometrial carcinoma. Cancer Res 52(6): 1622-1627

Kusama T, Miyagawa S, Moritan T, Kubo T, Yamada M, Sata H, Fukuta D, Matsunami K, Shirakura R (2003) Downregulation of NK cell-mediated swine endothelial cell lysis by DAF (CD55). Transplant Proc 35: 529-530

Li W, Geis N, Kirschfink M (2009) Specific targeting of anti-CD59 siRNA by Herceptin-conjugated liposomes improves complement-mediated cytotoxicity of breast carcinoma cell. Mol Immunol 46: 2830-2831

Li W, Szoka Jr FC (2007) Liquid based-nanoparticles for nucleic acid delivery. Pharma Res 24: 438-449

Macor P, Tripodo C, Zorzet S, Piovan E, Bossi F, Marzari R, Amadori A, Tedesco $\mathrm{F}$ (2007) In vivo targeting of human neutralizing antibodies against CD55 and CD59 to lymphoma cells increases the antitumor activity of rituximab. Cancer Res 67(21): 10556-10563

Morrison C, Zanagnolo V, Ramirez N, Cohn DE, Kelbick N, Copeland L, Maxwell GL, Fowler JM (2006) HER-2 is an independent prognostic factor in endometrial cancer: association with outcome in a large cohort of surgically staged patients. J Clin Oncol 24: 2376-2385

Mutch DG (2012) The more things change the more stay the same. Gynecol Oncol 124: 3-4

Rose PG (1996) Endometrial carcinoma. $N$ Engl J Med 335: 640-649

Santin AD, Bellone S, Gokden M, Palmieri M, Dunn D, Agha J, Roman JJ, Hutchins L, Pecorelli S, O'Brien T, Cannon MJ, Parham GP (2002) Overexpression of HER-2/neu in uterine serous papillary cancer. Clin Cancer Res 8: 1271-1279

Santin AD, Bellone S, Roman JJ, McKenney JK, Pecorelli S (2008) Trastuzumab treatment in patients with advanced or recurrent endometrial carcinoma overexpressing HER2/neu. Int J Gynaecol Obstet 102: $128-131$

Santin AD, Bellone S, Siegel ER, Palmieri M, Thomas M, Cannon MJ, Kay HH, Roman JJ, Burnett A, Pecorelli S (2005a) Racial differences in the overexpression of epidermal growth factor type II receptor (HER2/neu): a major prognostic indicator in uterine serous papillary cancer. $A m J$ Obstet Gynecol 192: 813-818

Santin AD, Bellone S, Van Stedum S, Bushen W, De Las Casas LE, Korourian S, Tian E, Roman JJ, Burnett A, Pecorelli S (2005b) Determination of HER2/neu status in uterine serous papillary carcinoma: Comparative analysis of immunohistochemistry and fluorescence in situ hybridization. Gynecol Oncol 98: 24-30

Santin AD, Bellone S, Van Stedum S, Bushen W, Palmieri M, Siegel ER, De Las Casas LE, Roman JJ, Burnett A, Pecorelli S (2005c) Amplification of $c$-erbB2 oncogene: a major prognostic indicator in uterine serous papillary carcinoma. Cancer 104: 1391-1397

Schwartz PE. The management of serous papillary uterine cancer (2006) Curr Opin Oncol 18: 494-499 
Siegel R, Ward E, Brawley O, Jemal A (2011) Cancer statistics, 2011: the impact of eliminating socioeconomic and racial disparities on premature cancer deaths. CA Cancer J Clin 61: 212-236

Slamon DJ, Leyland-Jones B, Shak S, Fuchs H, Paton V, Bajamonde A, Fleming T, Eiermann W, Wolter J, Pegram M, Baselga J, Norton L (2001) Use of chemotherapy plus a monoclonal antibody against HER2 for metastatic breast cancer that overexpresses HER2. $N$ Engl J Med 344: 783-792

Villella JA, Cohen S, Smith DH, Hibshoosh H, Hershman D (2006) HER-2/ neu overexpression in uterine papillary serous cancers and its possible therapeutic implications. Int J Gynecol Cancer 16: 1897-1902

Yarden Y, Sliwkowski MX (2001) Untangling the ErbB signalling network. Nat Rev Mol Cell Biol 2: 127-137

This work is published under the standard license to publish agreement. After 12 months the work will become freely available and the license terms will switch to a Creative Commons Attribution-NonCommercial-Share Alike 3.0 Unported License. 